\title{
TWITTER COMO FUENTE INFORMATIVA DE SUCESOS IMPREVISTOS: EL SEGUIMIENTO DE HASHTAGS EN EL CASO \#ARDEVALENCIA
}

\section{Twitter as an Information Source of Unexpected Events: Following Hashtags in the Case \#ArdeValencia}

\section{O Twitter como fonte informativa de sucessos imprevistos: o seguimento de hashtags no caso \#ArdeValencia}

LÓPEZ MERI, Amparo. Universidad Jaime I (España)

meri@uji.es

\section{Fecha de recibido: 12 de agosto de 2014}

Fecha de aceptado: 20 de octubre de 2014

\section{RESUMEN}

Las redes sociales se han integrado en las rutinas productivas de los periodistas. Se han convertido en otro altavoz para la difusión de contenidos y la interacción con el público. En cambio, en su papel de fuente informativa despiertan controversia. Para informarse, los profesionales suelen optar por la inmediatez de Twitter. El objetivo de este artículo es evaluar el contenido de los tuits asociados a hashtags, para calibrar su potencial como fuente informativa de sucesos imprevistos. Para este propósito, con las técnicas del análisis de contenido cuantitativo y cualitativo, se han analizado los tuits vinculados a \#ArdeValencia, etiqueta que alude a los incendios forestales de julio de 2012 en Valencia (España), que quemaron 50000 hectáreas. Las conclusiones indican que se difunde poca información exclusiva. Sin embargo, es frecuente la reiteración de opiniones y datos ya publicados en los medios tradicionales. Desde esta perspectiva, es cuestionable el valor de Twitter como fuente informativa, pero puede funcionar como termómetro del ambiente. Al respecto, casi la mitad de tuits contiene comentarios y críticas 
contra el Gobierno valenciano, por los recortes presupuestarios en prevención de incendios. En este sentido, se constata el uso del hashtag como herramienta de posicionamiento ideológico.

Palabras clave: periodismo, redes sociales, Twitter, hashtag, fuente informativa, periodismo ambiental, \#ArdeValencia.

\begin{abstract}
Journalists have integrated social networks in their professional routines. Social networks allow spreading content and interacting with the audience. However, in its role as an information source, they are controversial. Journalists often choose the immediacy of Twitter to get information. The aim of this paper is to analyze the content of tweets associated to hashtags to measure its potential as an information source of unexpected events. In order to do so, we have used a combination of quantitative and qualitative techniques of content analysis. We have studied the tweets linked to \#ArdeValencia, a tag about the wildfires in July 2012 in Valencia (Spain), which burned 50000 hectares. The findings indicate that almost no scoop is disseminated. We detect little exclusive information. Nevertheless, it's frequent to find reiterative comments, data and news previously published in the traditional media. From this perspective, Twitter's role as an information source is doubtful, but it can work as an ambiental thermometer. In fact, about half of tweets criticize the Valencian Government, due to their budget cuts on wildfire prevention. Therefore, we note users use hashtags to take sides and express their ideology.
\end{abstract}

Keywords: journalism, social networks, Twitter, hashtag, information source, ambiental journalism, \#ArdeValencia.

\title{
RESUMO
}

As redes sociais têm se integrado nas rotinas produtivas dos jornalistas. Têm-se convertido em outro alto-falante para a difusão de conteúdos e a interação com o público. No entanto, em seu papel de fonte informativa despertam controvérsia. Para se informar, os profissionais costumam optar pela imediatez do Twitter. O objetivo deste artigo é avaliar o conteúdo dos tweets associados a hashtags, para calibrar seu potencial como fonte informativa de acontecimentos imprevistos. Para este propósito, com as técnicas da análise de conteúdo quantitativo e qualitativo, se têm analisado os tweets vinculados a \#ArdeValencia, etiqueta que alude aos incêndios florestais de julho de 2012 em Valência (Espanha), que queimaram 50.000 hectares. As conclusões indicam que difunde-se pouca informação exclusiva. No entanto, é frequente a reiteração de opiniões e dados que têm sido publicados nos meios tradicionais. Desde esta perspectiva, é questionável o valor do Twitter como fonte informativa, mas pode funcionar como termómetro do ambiente. Ao respeito, quase a metade de tweets contém comentários e críticas contra o Governo valenciano, pelos recortes orçamentários em prevenção de incêndios. Neste sentido, constata-se o uso do hashtag como ferramenta de posicionamento ideológico. 
Palavras-chave: jornalismo, redes sociais, Twitter, hashtag, fonte informativa, jornalismo ambiental, \#ArdeValencia.

\section{Introducción}

Twitter se ha incorporado a las rutinas periodísticas en todas las fases del proceso de construcción del relato informativo. Se usa especialmente para la difusión y promoción de contenidos, pero también se consulta durante la cobertura de hechos noticiables, en busca de datos y contexto, y para conocer las opiniones de expertos y actores socialmente influyentes. Asimismo, se recurre a Twitter para pulsar el ambiente o estado de la cuestión sobre asuntos de relevancia. Al respecto, se debate si Twitter es una fuente informativa, pero todavía no existe una respuesta unánime. Parte de la profesión le reconoce este valor, especialmente para la cobertura de noticias de última hora, como revueltas o catástrofes naturales (Bradshaw, 2008; Farhi, 2011; Brown-Smith, 2012; Hu et ál., 2012; Vis, 2013; Hermida, 2013). Otra parte de la profesión se mantiene contraria o prefiere referirse a Twitter como fuente de pistas o alertas, pero no de información válida, pues cualquier dato difundido en la red social requiere ser contrastado (Herrero, 2012; Said et ál., 2013).

El seguimiento de hashtags o etiquetas es uno de los mecanismos empleados por los periodistas para informarse, porque cuando un suceso de actualidad despierta interés, suele generarse un hashtag para identificarlo. Con una simple búsqueda se puede acceder a todos los tuits que contengan dicha etiqueta. No todos los mensajes sobre el asunto incluirán el hashtag, pero sí una parte representativa. Algunos hashtags adquieren singular viralidad, lo que amplía el debate virtual y contribuye a localizar más cantidad de publicaciones.

El propósito de este artículo es evaluar el tipo de contenido que presentan los tuits, para calibrar el potencial de Twitter como fuente informativa en la cobertura de sucesos imprevistos. Este análisis se centra en los textos de los tuits, con independencia de si proceden de usuarios identificados o anónimos. Se valora si los mensajes de los tuits pueden incorporarse a las piezas informativas o usarse en alguna fase de su elaboración.

Para ahondar en la cuestión, se plantean dos objetivos específicos:

- Clasificar y cuantificar el contenido de los textos de los tuits sobre un suceso de actualidad imprevisto, para determinar qué tipo de contenido predomina.

- Evaluar los significados de los textos, para valorar el grado de exclusividad y diversidad de las informaciones y opiniones relacionadas con el suceso.

En relación con los citados objetivos, se parte de tres hipótesis:

- Los usuarios de hashtags asociados a sucesos imprevistos tienden a opinar y posicionarse ideológicamente más que a informar.

- El porcentaje de información exclusiva es escaso, frente al elevado volumen de noticias ya publicadas en medios de comunicación tradicionales, lo que resta valor a Twitter como fuente informativa y la convierte en caja de resonancia de contenidos ya publicados. 
- La reiteración de contenidos es habitual y disminuye la diversidad de los significados en circulación, aunque contribuye a generar un determinado ambiente o estado de la cuestión.

\section{La gestión de fuentes informativas en el entorno digital}

\subsection{Un escenario de transformación en la gestión de fuentes}

Los medios juegan un rol determinante en la formación de la opinión pública (Grossi, 2007), porque los ciudadanos han aceptado la mediación de los periodistas para entender el mundo. Las noticias atribuyen significados y sentido a los acontecimientos (Casero-Ripollés, 2009), y el uso eficiente de fuentes garantiza el derecho a estar correctamente informado en sociedades democráticas (Wolf, 1994). La irrupción de Internet está cambiando esta relación de mediación simbólica, pues permite la proliferación de medios y rompe el monopolio informativo de las fuentes oficiales. A través de las redes sociales, ciudadanos y colectivos hasta ahora marginados pueden tener voz (Castells, 2009; Casero-Ripollés \& Feenstra, 2012).

Las nuevas tecnologías han transformado las fases de producción, distribución y consumo de contenidos informativos. Respecto a la producción, destaca la sobreinformación, que puede derivar en desinformación, superficialidad y falta de reflexión (Serrano, 2009 \& 2013), especialmente cuando impera la lógica digital de la velocidad. En paralelo, no obstante, algunos medios tratan de diferenciarse y apuestan por formatos de mayor calidad, que implican más investigación y análisis (García-Avilés et ál., 2014). Estas tendencias exigen periodistas polivalentes, capaces de asumir diferentes funciones y de adaptar sus creaciones a múltiples formatos y soportes (Edo \& Mayoral, 2011; Ortells-Badenes \& González-Molina, 2012).

Respecto a la difusión, los medios disponen de versión online y oferta a la carta. Comparten sus contenidos en las redes sociales y a través de sus aplicaciones para dispositivos móviles. El consumo de información se realiza en cualquier parte, desde el teléfono, la tableta o el ordenador portátil. En todas las redacciones, con independencia del grado de convergencia digital, la participación del público se está convirtiendo en una cuestión estratégica (García-Avilés et ál., 2014), lo que requiere una mejora de la competencia interactiva de los periodistas. Parece haber consenso en que el futuro de los grupos mediáticos pasa por diseñar una gestión común de los medios sociales y reforzar la motivación hacia el contenido generado por los usuarios (García-Avilés et ál., 2014). Sin embargo, la realidad es otra. Los medios todavía están lejos de abrir la conversación y crear vínculos con sus receptores (López \& Alonso, 2013). Se limitan a enlazar noticias colgadas en línea previamente y apenas responden mensajes (Requejo \& Herrera, 2014). Comprenden el potencial de las redes sociales, pero aún están en una fase incipiente de aprovechamiento (Flores, 2009), en parte, por la falta de cultura profesional y empresarial, pues muchos periodistas siguen mediados por modelos tradicionales ajenos a escenarios digitales (Said et ál., 2013).

Frente a la crisis económica y al vértigo en las redacciones, la coyuntura ofrece una oportunidad para revalorizar la profesión. Todavía sin un modelo claro de negocio (Casero-Ripollés, 2010), la salida podría residir en la oferta de contenidos más elaborados, reflexivos y analíticos (Micó, 2011; García-Avilés et ál., 2014), por los que el público estaría dispuesto a invertir. Actualmente, el público es reacio al pago por noticias online. La solución podría consistir en enriquecer la oferta de información con otros servicios de entretenimiento complementarios 
(Goyanes, 2014). En España, algunos medios digitales, como Eldiario.es o La Marea, ensayan fórmulas de crowdfunding y viven de los ingresos de sus socios.

Cuestión distinta es que la multiplicación de medios, canales y contenidos que propicia Internet se traduzca en pluralidad. De hecho, se constata el dominio de voces oficiales e interesadas (García, 2010; Casero-Ripollés \& López-Rabadán, 2013). No es un fenómeno reciente. Los periodistas han contribuido durante décadas a sustentar este status quo privilegiado para las fuentes cercanas al poder (Gans, 1980; Tuchman, 1983), al marginar a colectivos minoritarios. Los políticos, por su parte, siguen tratando de controlar a los medios (Soengas 2007; Casero-Ripollés, 2009). La aparente pluralidad que ofrece Internet se torna en homogeneidad, porque los medios beben de las mismas fuentes. Por eso, la función de mediación puede devenir en manipulación y superficialidad (Serrano, 2009; 2013). Colectivos ciudadanos y partidos políticos minoritarios, escépticos ante el riesgo de una mediación condicionada, recurren a las redes sociales para dirigirse directamente a la audiencia. En paralelo, adquiere fuerza la corriente que reivindica la correcta gestión de fuentes como criterio profesional de calidad, con estudios que cuestionan la independencia de los medios en su control al poder político (Franklin, 2011).

\subsection{Redes sociales y nuevos actores: ventajas y riesgos}

Las redes sociales contribuyen a expandir el oficialismo de las fuentes, porque las instituciones usan estas plataformas para sus intereses. En el debate virtual, también se reproducen los esquemas de poder tradicionales (Deltell et ál., 2013), especialmente cuando el volumen de publicaciones es inabarcable, lo que favorece que los usuarios se limiten a seguir a las fuentes que ya conocen fuera del espacio público virtual. No obstante, al mismo tiempo, las redes sociales democratizan el proceso de comunicación (Castells, 2009), con la entrada de nuevos actores y el acceso a una gran cantidad de información, lo que redunda en mayor transparencia (Della Porta, 2011). El público no solo observa, sino que participa y se integra cada vez más en el proceso de construcción de las noticias (Orihuela, 2011).

En España, por ejemplo, el movimiento 15M ha logrado colarse en la agenda temática de los medios y en el debate político gracias al uso de redes sociales (Casero-Ripollés \& Feenstra, 2012). El activismo online del 15M, imitado por otros colectivos ciudadanos, como la Plataforma de Afectados por la Hipoteca (PAH) o Juventud Sin Futuro (JSF), contribuye a mejorar la infraestructura de estas organizaciones, optimizar recursos e imponer mensajes propios, que de otra manera no llegarían al público (Castells, 2012; Micó \& Casero-Ripollés, 2013). Internet y las redes sociales constituyen plataformas ideales para el escrutinio público como manifestación de activismo político (Feenstra \& Casero-Ripollés, 2014). En este sentido, la monitorización que realizan los ciudadanos en los medios sociales, para vigilar, filtrar información y difundir puntos de vista alternativos, "es una forma de contrapoder que desafía a los centros de poder político y económico” (Feenstra \& Casero-Ripollés, 2014, p. 2462).

Las redes sociales ponen en contacto a periodistas y potenciales suministradores de contenidos. Es fundamental la relación triangular que se entabla entre periodistas, fuentes y usuarios (Broersma \& Graham, 2012). En gran medida, la calidad de los contenidos informativos dependerá de la credibilidad que los periodistas confieran a los perfiles a los que asignan el papel de fuentes. El crowdsourcing o creación colaborativa de contenidos responde a esta idea y a la concepción de las redes sociales como "enormes fuentes de inteligencia colectiva", de las que el periodista puede sacar partido (Broersma \& Graham, 2012, p. 404). No obstante, esta relación triangular puede 
quedar mutilada si las fuentes eliminan la mediación de los periodistas y se dirigen directamente a otros actores, sin filtros (Rubio García, 2014). De momento, en el caso de los políticos, no se ha comprobado que su activismo en las redes modifique las inclinaciones del electorado, al menos en España (Abejon et ál., 2012).

En el lado positivo, la construcción colaborativa de noticias fomenta la cultura de la participación propia de la Web social. También puede entrañar peligros, como la propagación de rumores, si el periodista no verifica la información que circula por Internet. La literatura sobre esta materia reivindica un reenfoque del papel del periodista, para que ejerza de guardián de la información en las redes sociales (Bruno, 2011; Hermida, 2012). Según explica Hermida (2012), el periodista deberá justificar en la propia red cómo se construye la notica, cómo se impugna, deniega o verifica cada fragmento de información. El periodismo, bajo este prisma, ya no consiste en presentar productos acabados, sino en el proceso de construirlos, evaluarlos y examinarlos, en tiempo real y en público.

Asimismo, se defiende la especialización del periodista como content curator o curador de contenidos (Newman 2009; Bhargava, 2009; Bruno, 2011; Malini, 2011; Hermida, 2012; McElroy, 2013; Cappeletti, 2014; Bakker, 2014). En un mundo controlado por flujos de información, dice Bhargava (2009), es fundamental una selección personalizada, de calidad y continua, del mejor contenido acerca de temas específicos. Este autor considera que el futuro de la Web social será impulsado por los curadores de contenidos, como editores encargados de recoger y compartir contenidos online para el consumo de otros. La cronología de actualizaciones o timeline de una red social puede ser el espacio ideal para ejercer la curaduría de contenidos (Malini, 2011).

\section{La naturaleza de Twitter y su impacto en el periodismo}

Los investigadores en el ámbito del periodismo reconocen, con relativo consenso, que Twitter es algo más que una red social (Romero et ál., 2011; Bruns \& Burgess, 2012; Hermida, 2013). Diferentes estudios constatan que el fundamento del vínculo entre sus usuarios está en el intercambio de información, y no en las relaciones sociales entabladas en plataformas como Facebook (Kwak et ál., 2010). En esta línea, Bruns y Burgess definen Twitter como un híbrido entre "social networking site" (red social) y "ambient information stream" (corriente de información ambiental), porque combina las prácticas esenciales de las redes sociales, tales como el following (seguir a otros) o el friending (hacer amigos), con la esencia del broadcasting o difusión de contenidos. Esta convergencia sería la que confiere a Twitter importancia para el periodismo (2012, p. 3).

En Twitter coexisten dos redes independientes, que cumplen funciones distintas (Bruns \& Burgess, 2012). Por una parte, se crea la red de followers (seguidores) y followees (seguidos), mediante relaciones duraderas. El número de seguidores mide la popularidad del usuario como fuente para otros actores, mientras que los perfiles que se siguen miden el volumen de noticias a las que se expone cada actor. En este sentido, los usuarios se convierten en gatekeepers, puesto que pueden confeccionar su propia lista de medios y periodistas a los que seguir, y consumir su propia programación. Pero además de consumidores, con sus publicaciones y recomendaciones, se convierten en soportes de contenidos para sus seguidores (Rodríguez \& García, 2013).

Por otro lado, se entablan redes esporádicas en torno a temas de interés compartido. Estas relaciones temporales están coordinadas por un hashtag o etiqueta, de vida relativamente corta y de origen espontáneo. Mediante las etiquetas se comparten argumentos con perfiles ajenos a la red permanente de seguidores. En este ámbito, nacen tantas relaciones como asuntos interesen a los usuarios. Estas relaciones finalizan cuando pierde vigor el 
debate compartido y deja de usarse el hashtag. El hashtag funciona como nexo de búsqueda y coordinación de contenidos. Permite clasificar y organizar tuits. Es una convención creada orgánicamente por la comunidad de usuarios para agregar mayor nivel de contexto y metadata a las conversaciones (Rojo, 2012). Se emplean para buscar información y opiniones, y también para expresar posturas, lo que remarca que el objetivo en Twitter es el debate, no las relaciones interpersonales (Bruns \& Burgess, 2012).

Los periodistas están normalizando el uso de Twitter y encajándolo en sus rutinas profesionales, pero también están modificando algunas de sus normas y prácticas debido a Twitter (Farhi, 2009; Larsosa et ál., 2012; Newman et ál., 2012; Carrera Álvarez et ál., 2012; Oriella PR Network, 2012; 2013). En general, usan Twitter para informar, pero también retuitean y enlazan las aportaciones de otros usuarios. Por eso, además de fuentes, sus cuentas funcionan como soportes informativos. Ejercen de gatekeepers para sus lectores. Además, cuando tienen muchos seguidores, suelen introducir comentarios y valoraciones personales (Larsosa et ál., 2012; Rodríguez \& García, 2013).

Como se ha adelantado en el epígrafe anterior, los medios todavía son conservadores. Sus cuentas corporativas funcionan como canales RSS o redistribuidores de información (López \& Alonso, 2013), para su promoción (Noguera, 2013), con enlaces que redirigen el tráfico hacia sus páginas web, sin propiciar la conversación colectiva, ni crear comunidades activas (Salaverría et ál., 2010; Greer \& Ferguson, 2011; García de Torres et ál., 2011; Said et ál., 2013; Requejo \& Herrera, 2014). Los periodistas, en cambio, se muestran más creativos e interactúan con sus seguidores (Larsosa et ál., 2012; Artwick, 2013), incluso evitan citar en sus perfiles la empresa en la que trabajan (Rodríguez \& García, 2013), como prueba de independencia. Los periodistas que laboran para medios de referencia, suelen conseguir mucha audiencia gracias a su filiación laboral (Larsosa et ál., 2012). Se intuye que los periodistas independientes o de medios locales habrán de ingeniárselas de otro modo para aprovechar el potencial de Twitter, llamar la atención y captar seguidores.

Los periodistas también buscan información y contactan con fuentes en Twitter (Carrera et ál., 2012). Desde esta perspectiva, cualquier usuario, potencialmente, puede colaborar en la elaboración de contenidos. Este planteamiento, sin embargo, genera críticas. Los propios periodistas advierten de la imposibilidad de verificar tanta información y del peligro de publicar rumores o datos manipulados (Phillips, 2010; Hermida, 2010). Este obstáculo podría salvarse igual que en los medios convencionales, si se somete a los usuarios-fuente a un proceso de autentificación (Hermida, 2010; 2012). Al respecto, en España, el 90\% de los periodistas con perfil en Twitter asegura que contacta con las fuentes originales de la información, y el $84 \%$ realiza búsquedas en Internet para identificar a los usuarios-fuente (Carrera et ál., 2012).

\subsection{El periodismo ambiental en Twitter}

Frente a la corriente que estudia Twitter en el marco del periodismo ciudadano (Deuze, 2005; Niles, 2007), otra línea de investigación concibe esta red social como un sistema de comunicación autónomo, inspirado en el concepto de periodismo ambiental (Hermida, 2010; Burns, 2010). En su teorización de la noción de periodismo ambiental, Hermida asegura que Twitter, como servicio de microblogging, constituye un "sistema de conciencia informativa" colectivo, que nace de la conexión con otros usuarios, y que aporta a los periodistas "formas más complejas de comprensión en las sutilezas de la comunicación pública” (2010, p. 300). 
Esta conciencia informativa se forma, desde la perspectiva del periodismo ambiental, porque el valor de la comunicación no se encuentra en cada tuit, sino en la suma, en el retrato mental creado por un número determinado de mensajes en un período de tiempo concreto (Hermida, 2010). A través de Twitter se difunden y comparten noticias online, con mensajes cortos, rápidos y frecuentes. Pretender procesar cada fragmento de información resulta un objetivo inalcanzable. Sin embargo, puede ser útil detectar tendencias en la combinación de tuits. Frente al saber fragmentado e individual, la suma enriquece el proceso de formación de conocimientos. La toma de conciencia informativa y la inteligencia colectiva, que surgen de la combinación de mensajes, mejoran la comprensión de los periodistas (Hemida, 2010; 2013).

En esta línea, Burns (2010) establece un paralelismo entre el ambiente de Twitter y la música ambiental, que suena de fondo y puede pasar desapercibida, a menos que haya un cambio en el tono o el estilo. Lo mismo ocurre en Twitter. En un debate sobre un tema de actualidad, los mensajes individuales pasan a un segundo plano, pero el cambio de tono o enfoque en un número significativo de tuits llamará la atención del usuario (Bruns \& Burgess, 2012).

Sobre el valor del ambiente en Twitter, en las últimas elecciones presidenciales de Brasil, se detecta que los intereses de los ciudadanos se apartan del mensaje oficial. Nunomura (2013) constata la falta de correspondencia entre la agenda de los medios y las opiniones expresadas por los usuarios. Al respecto, compara las críticas de los ciudadanos con la labor de los observatorios públicos de la prensa. Sin embargo, sostiene que hacen falta más estudios para comprobar si Twitter funciona como un barómetro de la opinión pública, aunque percibe indicios favorables. Según apunta, los ciudadanos quieren opinar, pero también decidir qué temas popularizar y debatir (Nunomura, 2013).

No obstante, en España, un estudio reciente sobre Twitter y la teoría de la Agenda-Setting concluye que existe una clara correspondencia entre la agenda del público y la establecida por los medios de comunicación tradicionales (Rubio, 2014). Los asuntos comentados en Twitter suelen explicarse también desde los medios, aunque "esta correlación de agendas no siempre depende de la influencia de los medios en la opinión pública" (Rubio, 2014, p. 262). La similitud suele deberse a que medios y usuarios tienen un acceso semejante a la información. Excepcionalmente, en la línea apuntada por Nunomura (2013), se detecta que los usuarios de Twitter también publican información basándose en su propia experiencia y conocimientos, y no en los contenidos difundidos previamente por los medios de comunicación (Rubio, 2014).

\subsection{El hashtag como herramienta para el gatekeeper}

El hashtag es una herramienta de gran utilidad para el gatekeeper y los profesionales encargados de coberturas informativas. Se trata de una etiqueta precedida del símbolo de la almohadilla que cualquier usuario puede crear para identificar un asunto. Su perdurabilidad depende de la comunidad, porque si no se reutiliza, no existe. El hashtag propicia la formación de una comunidad ad hoc, que debate o conversa sobre el tema etiquetado.

La literatura sobre Twitter define el hashtag como elemento estructurador y coordinador de contenidos (Bruns \& Burgess, 2012). Se tiende a delimitar sus funciones, usos y gratificaciones, como mecanismo de participación en el debate de la actualidad informativa, y también en relación con el periodismo ambiental (Brown-Smith, 2012). El hashtag facilita la búsqueda de contenidos, aunque procedan de actores anónimos o desconocidos, especialmente cuando se trata de cuestiones de última hora, a las que se accede rápidamente gracias a la etiqueta. En 
esta línea, el hashtag es fuente de contenidos tematizados. Esta visión del hashtag apoya la concepción de Twitter como plataforma de diseminación y discusión (Bruns \& Burgess, 2012; Brown-Smith, 2012). Si generan retuits, contribuyen a la cohesión de la comunidad ad hoc y a la propagación de contenidos. En ocasiones, el hashtag se utiliza para dar más visibilidad al tuit, sin intención de entablar conversación.

Además, debido a sus características, el hashtag es compatible con la función de la curaduría de contenidos (Abbagliati, 2010), pues incluir la etiqueta durante un debate o conversación, supone seleccionar o recomendar el comentario al resto de participantes o interesados. Emplear el hashtag como marcador de tuits puede contribuir al gatekeeping colaborativo de contenidos, una práctica que los usuarios ya ensayan en la prensa digital (Singer, 2011). Según argumenta Malini (2011), la nueva cultura de la producción y el consumo de información, que se materializa en el timeline o cronología de tuits, depende de la comunidad. En Twitter, cada usuario es una comunidad de autores, cuyo alcance se mide por el número de respuestas, menciones, comentarios y retuits que logra (Malini, 2011). En este contexto, el hecho noticioso pasa a ser un bien común (Shirky, 2011), que trasciende al producto físico generado por los medios de comunicación.

\section{Metodología}

\subsection{Técnica}

Esta investigación se aborda mediante las técnicas del análisis de contenido cuantitativo y cualitativo, que permiten, a partir de los resultados obtenidos, formular inferencias generalizables (Krippendorff, 1990), para la interpretación de los mensajes de los tuits. Estas técnicas contribuyen a desentrañar el significado manifiesto de los mensajes (Bardin, 1996), en este caso de los tuits ligados a hashtags de actualidad.

El modelo de análisis incluye el diseño de once categorías para clasificar los contenidos que se difunden en los tuits:

Información conocida: datos contrastados con fuentes, exentos de valoraciones, normalmente publicados en medios de comunicación.

Información exclusiva: datos no publicados previamente en otros foros o medios de comunicación, aportados por actores que los conocen en calidad de testigos o fuentes. Se considera información exclusiva la que procede de blogueros independientes, aunque los datos también estén disponibles en sus blogs. Se excluye la información obtenida en actos públicos de acceso libre, como comparecencias en el Congreso televisadas, pues no es necesario recurrir a Twitter para conocer su contenido.

Interpretación: explicaciones o juicios de los hechos, acompañados de datos o argumentos que los sostienen.

Opinión personal: supuestos en los que el actor expresa sus sentimientos y deseos, narra sus vivencias o emite valoraciones personales exentas de críticas al Gobierno o a la clase política.

Opinión crítica: valoraciones expresamente emitidas para cuestionar al Gobierno y a los miembros del Gobierno, y también para poner en duda actuaciones concretas de políticos o partidos políticos. Se incluyen también las críticas a la monarquía, las instituciones públicas y los medios de comunicación. 
Promoción: mensajes que ensalzan a otro usuario, sus productos, actividades o iniciativas. Se incluyen también los mensajes de autopromoción, que enaltecen al propio usuario, al colectivo al que pertenece o a sus compañeros, o que ponen en valor los productos, actividades o iniciativas promovidas por los mismos.

Humor: expresiones irónicas o satíricas y bromas.

Insulto: descalificaciones, insultos y palabras malsonantes.

Ruido: supuestos en los que el contenido no está vinculado al suceso imprevisto aludido por el hashtag.

Sin texto: supuestos en los que el tuit no contiene texto, aunque sí otros elementos, como enlaces, menciones o hashtags.

Otro: aspectos no incluidos en ninguna de las categorías anteriores, como las interacciones con los seguidores.

La mayoría de categorías son combinables. Es decir, un mismo tuit puede estar clasificado y contabilizado en varias categorías a la vez, por ejemplo, si su texto combina información conocida y opinión personal. Por eso, en el apartado dedicado a la exposición de resultados, la suma de porcentajes de la gráfica 1 supera el 100\%. No obstante, el porcentaje de cada tipo de contenido representa su peso real respecto al total de la muestra. Por último, aunque no es objeto de este estudio detallar la autoría de los tuits, se han establecido dos categorías para identificar a los usuarios. Se habla de usuarios anónimos, cuando ha resultado imposible verificar su identidad, y de usuarios identificados. En este segundo grupo se incluyen profesionales de diversa índole, como políticos, partidos políticos, medios de comunicación, periodistas, empresas, sindicatos y colectivos ciudadanos. Como sinónimos de usuario, se recurre a los términos actor, perfil y cuenta. Como sinónimo de tuit, se emplean los términos publicación, mensaje y actualización.

\subsection{Muestras}

Para este análisis se ha seleccionado el hashtag \#ArdeValencia, etiqueta vinculada a los incendios de julio de 2012 en la provincia de Valencia (España), que quemaron 50000 hectáreas en los términos muncipales de Cortes de Pallás y Andilla. En concreto, en este artículo se analizan todos los tuits publicados durante cinco días, desde el 7 hasta el 11 de julio, que ascienden a un total de 491 mensajes. Las fechas seleccionadas coinciden con la fase final del debate sobre el suceso, que derivó en contundentes críticas al Gobierno valenciano, por los recortes presupuestarios en efectivos para la lucha contra incendios y por la gestión de las labores de extinción, en las que falleció el piloto de un helicóptero.

Cada tuit constituye una unidad de análisis, a la que se aplica el modelo descrito en el epígrafe anterior. El estudio se centra en los textos de los tuits, con independencia de su autoría. Se excluyen los enlaces, fotografías, vídeos y otros elementos o archivos adjuntos a los tuits. La recopilación de unidades se ha capturado con la aplicación informática Tweet-Tag, que monitoriza hashtags con un margen de error del 0,77\%. Se excluyen los retuits (reenvió de tuits ya publicados), pues para el objetivo de esta investigación, que es evaluar el tipo de contenido difundido en relación a un hashtag, se ha considerado suficiente analizar una sola vez cada texto original. Los retuits, por ser repeticiones, amplifican los datos y comentarios, pero no añaden novedades respecto al tipo de contenido. 


\section{Resultados del análisis de \#ArdeValencia}

\subsection{La distancia entre la información exclusiva y la opinión crítica}

Tras analizar el contenido de los 491 tuits de la muestra, se observa que predominan las opiniones, pues críticas y valoraciones personales están presentes en el $46,6 \%$ de los textos (229 tuits). Por detrás, los contenidos informativos ocupan el $39 \%$ de los mensajes (191 tuits), conforme a la gráfica 1 . El resto de contenidos queda a mucha distancia, como la interpretación, prácticamente inexistente (1,4\%); la promoción (9\%) o el humor (4\%). También se registran, muy esporádicamente, insultos (2,2\%) y tuits sin texto (2,6\%). El ruido deriva del $8,3 \%$ de los textos, y se corresponde con ideas ajenas a los incendios de Valencia, que no aportan valor a los periodistas encargados de cubrir esta noticia. Por último, se incluye la categoría Otro (8,7\%), que alude a otro tipo de significados, normalmente interacciones con el público.

\section{Gráfica 1. El contenido de los textos de los tuits sobre \#ArdeValencia}



Fuente: elaboración propia.

En relación con las informaciones, destaca la escasez de datos exclusivos, solo en el 2,8\% de los textos (14 tuits), como refleja la gráfica 2. Además, estos datos exclusivos no suelen ser relevantes y van acompañados de opiniones personales o mensajes promocionales. Por el contrario, es muy habitual aprovechar el hashtag \#ArdeValencia para difundir información conocida, es decir, publicada previamente en medios de comunicación o blogs. Así ocurre en el $36 \%$ de los textos (177 tuits), que redifunden titulares de prensa sobre el número de hectáreas quemadas, los daños al medioambiente y las indemnizaciones aprobadas por el Gobierno valenciano para los damnificados. 
Gráfica 2. Desglose del tipo de informaciones y opiniones



Fuente: elaboración propia.

Respecto a las opiniones, dominan las críticas, en el 29,7\% de los mensajes (146 tuits), frente a las valoraciones personales, en el $17 \%$ (83 tuits). Entre las críticas, se exigen responsabilidades políticas y se cuestiona la labor del Gobierno valenciano, gobernado por el Partido Popular (PP). También se discuten las prioridades de los líderes del PP y se denuncian aspectos como la deficiente gestión en las labores de extinción, la escasez de medios y los recortes presupuestarios para la prevención de incendios. En cambio, las opiniones personales, exentas de crítica al Gobierno y los políticos, consisten en la expresión de deseos y sentimientos. Los actores empatizan con los vecinos de las zonas afectadas por el fuego, como se aprecia en el siguiente tuit del colectivo ciudadano @Fotomovimiento.

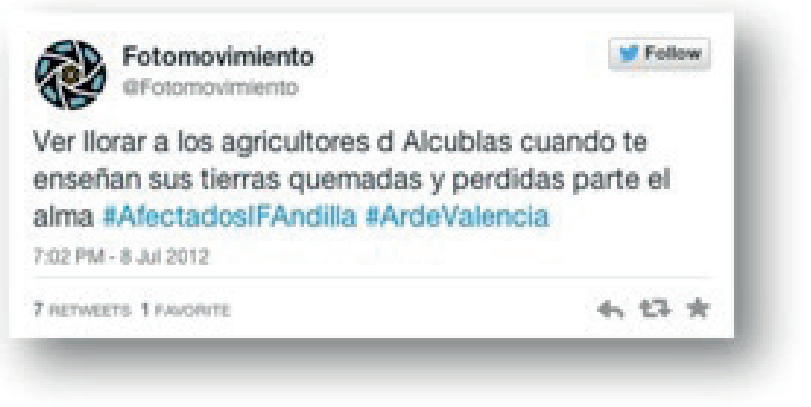

También se comprueba que los usuarios retuitean más opiniones que informaciones. En este sentido, el $35 \%$ de los tuits que difunden críticas y/o valoraciones personales logra algún retuit. El porcentaje disminuye al $23 \%$ si los mensajes son informativos. Si se atiende a la suma total de retuits, la diferencia todavía es más pronunciada. Los tuits con opiniones logran 373 retuits, frente a los 170 retuits que consiguen las actualizaciones con información.

Además, se constata la costumbre de reproducir textualmente el titular de los enlaces que acompañan a los textos de los tuits. Esta práctica se detecta en el $16,3 \%$ de las publicaciones (80 tuits). Normalmente, se trata 
de titulares de prensa, pero también se incluyen títulos de entradas de blog. Solo la cuarta parte de estos titulares incluye opinión. Lo habitual es que sean titulares informativos (48\%), como muestra el tuit del periodista @pau_sempere.

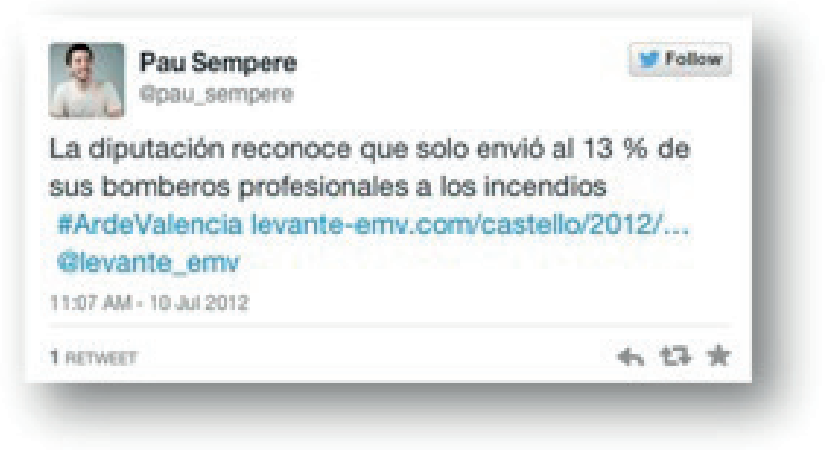

En general, se constata que la tendencia a copiar textos de otros actores es común a cuentas que optan por informar. En cambio, se añaden ideas propias cuando se manifiestan opiniones. En ocasiones, las publicaciones combinan diferentes tipos de contenido. A modo de ejemplo, el 4,27\% de los mensajes ( 21 tuits) compagina información con opinión crítica, mientras que el 1,6\% suma opiniones críticas y valoraciones personales ( 8 tuits), y otro $1,6 \%$ mezcla información exclusiva con valoraciones.

\subsection{Los significados asociados a los tuits informativos y valorativos}

Desde una perspectiva cualitativa, interesa centrar el análisis en los significados que transmiten los tuits informativos y valorativos asociados a \#ArdeValencia, con el objetivo de evaluar el grado de diversidad. Respecto a las informaciones, proceden en su mayoría de noticias publicadas en medios de comunicación y blogs de expertos o usuarios anónimos. Los actores, además, suelen mencionar a las fuentes. En la mayoría de ocasiones, se resumen titulares de prensa o directamente se copian de forma textual. Los principales contenidos informativos se articulan en torno a siete ejes:

- Políticas de prevención de incendios y gestión de la extinción: envío de pocos efectivos y eliminación de la figura de la brigada forestal de verano en 31 municipios.

- Consecuencias de los incendios: 50000 hectáreas quemadas que tardarán 100 años en recuperarse, 1000 millones de euros en pérdidas, más de 100 especies de mamíferos y aves afectadas, riesgo de desertización en el área calcinada y peligro de nuevos incendios por la mala gestión agroambiental.

- Respuestas del Gobierno valenciano: concesión de ayudas a los damnificados por valor de 9 millones de euros, presentación de un proyecto a la Unión Europea, para la reducción del impacto de incendios, y modificación de la legislación urbanística, para la recalificación de zonas forestales quemadas. También se explica que el presidente del Gobierno valenciano no comparecerá para explicar las actuaciones de extinción. 
- Creación de una plataforma de personas afectadas por la pérdida de sus casas y terrenos, como se observa en el tuit publicado por el profesor de Educación Secundaria @ximoberna. Se informa de las asambleas y reuniones de la plataforma, que reivindica más ayudas económicas por los daños.

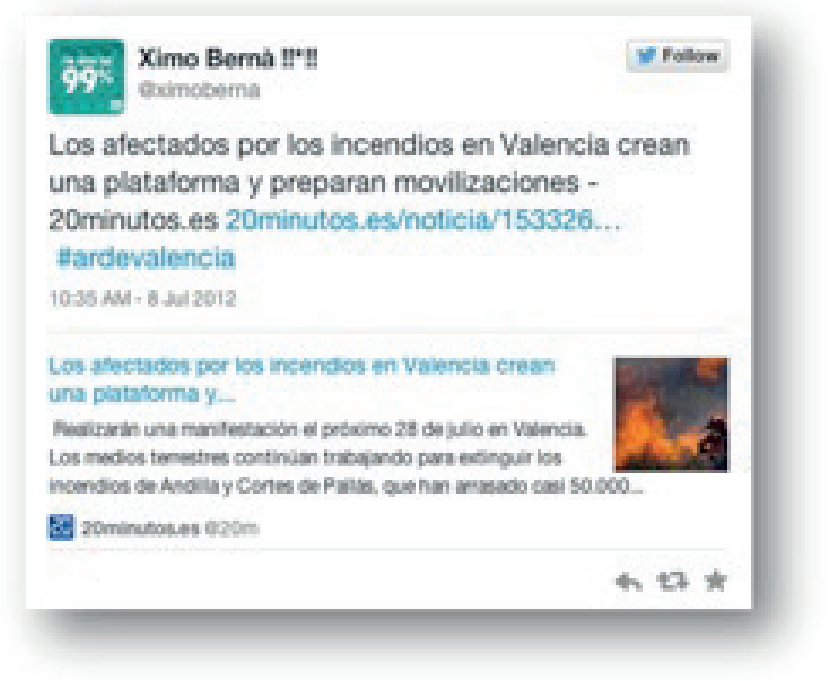

- Comparecencia del ministro de Agricultura y Medio Ambiente en el Congreso de los Diputados de Madrid, para explicar el recorte de efectivos realizado por el Gobierno valenciano, e informar de las labores de extinción.

- Propuestas de los partidos políticos de la oposición para cambiar las políticas de desarrollo rural.

- Nuevo incendio forestal en La Marina, comarca de la provincia de Alicante.

La lista de temas es breve si se compara con el total de tuits (491 unidades). Se concluye que las mismas informaciones se repiten constantemente. Cada día analizado cuenta con un par de titulares que copan la atención de los participantes en el debate.

Respecto a la difusión de información exclusiva, no publicada con anterioridad en medios de comunicación, es simbólica y de escasa relevancia. Guarda relación con el nuevo incendio de Alicante y las acciones de la plataforma de damnificados. En el 35,7\% de los casos, la información exclusiva procede de actores anónimos, como @_lybed y@Cantavestrella, que publican las siguientes actualizaciones. 
En el ámbito de las opiniones críticas, se aprecia la misma tendencia citada en relación a las informaciones. Son abundantes, pero reiterativas. Pueden organizarse en torno a tres pilares:

- Críticas al Gobierno valenciano, presidido por Alberto Fabra (PP). Se denuncian los recortes económicos y la disminución de brigadas forestales. Se exige la dimisión del consejero de Gobernación, Serafín Castellano, responsable de las políticas de prevención de incendios forestales. También se acusa al Ejecutivo valenciano de corrupción, por modificar la legislación, presuntamente, para favorecer la recalificación y construcción en terrenos quemados por incendios forestales. Estos tuits difundidos por el periodista@Fpiera y el usuario anónimo @josepc4 son ejemplos de crítica. 

Ios \#recortes el mismo gobierno tiraba dinero a saco en brigadas inútiles \#ArdeValencia

12:00 AM - 9 Jul 2012

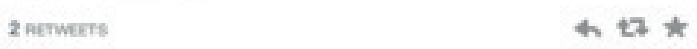

\section{7. josepc4}

Al PP no lo entiende ni dios, recortan en efectivos (d 160 a $26 . .$.$) para incendios y ahora presumen de$ ayudas a damnificados \#ArdeValencia

$11: 58$ AM - 7 Jul 2012

- Críticas al presidente del Gobierno español Mariano Rajoy (PP), por no visitar los municipios afectados por los incendios. Los usuarios aprovechan el hashtag \#ArdeValencia para rechazar las prioridades de Rajoy, que en lugar de acudir a Valencia, se desplaza a Galicia, donde se ha recuperado una obra religiosa, el Código Calixtino. También se cuestionan los recortes en prestaciones sociales y la subida de impuestos como el IVA.

- Críticas genéricas al Partido Popular, sin centrarse en ningún político en particular.

Gráfica 3. Clasificación de las opiniones críticas vinculadas a \#ArdeValencia

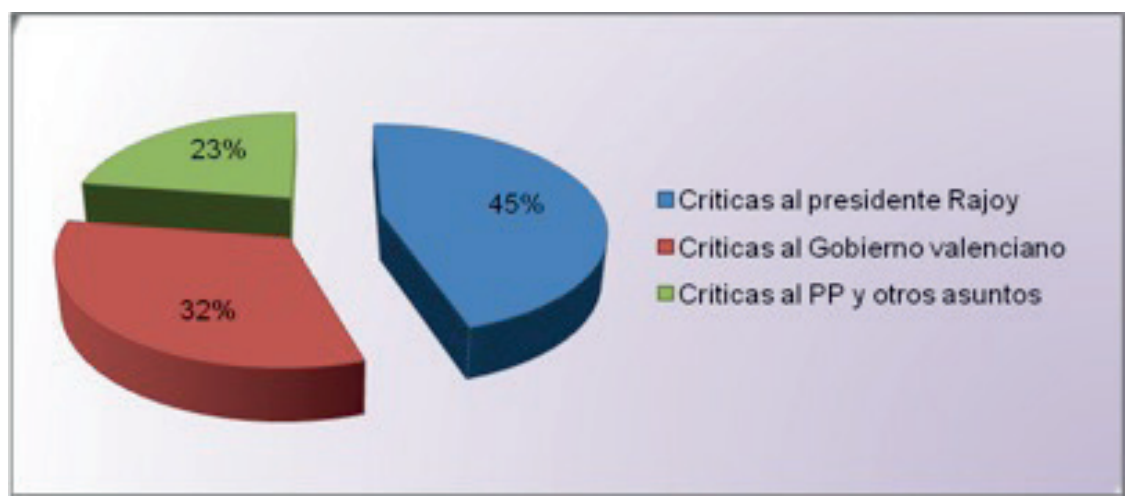

Fuente: elaboración propia. 
Como sucede con las informaciones, cada día se detecta un tema de crítica que adquiere fuerza y se propaga a gran velocidad:

- El 7 de julio de 2012 se insiste en el recorte de efectivos contra incendios y se culpa al Gobierno valenciano, como apunta este tuit anónimo de @LuisWSev.

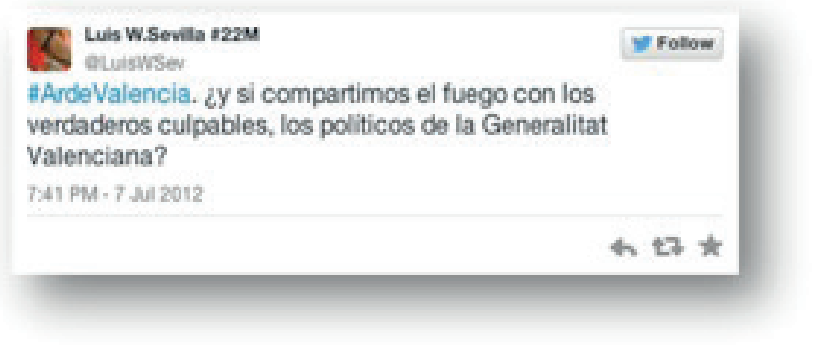

- Los días 8 y 9 de julio se recrimina la ausencia de Mariano Rajoy (PP) en las zonas arrasadas por el fuego. En este sentido, el presidente del Gobierno protagoniza el $45 \%$ de los tuits que contienen alguna crítica, como se aprecia en el siguiente ejemplo del periodista @juliovicedo.

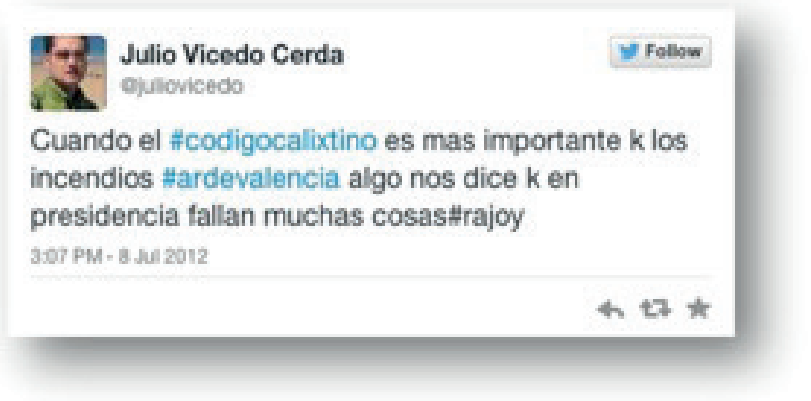

- El día 10 de julio se exigen respuestas y responsabilidades, porque el PP vota en contra de la comparecencia del presidente del Gobierno valenciano, Alberto Fabra (PP), para dar explicaciones. Entre otros actores, lo denuncian los partidos de la oposición. Es el caso de @SocialistesVal, la cuenta del Partido Socialista (PSOE) en Valencia. 
- El día 11 se opina sobre la intervención en el Congreso de los Diputados de Madrid del ministro de Agricultura y Medio Ambiente, que entonces era Miguel Arias Cañete (PP), actualmente eurodiputado en Bruselas.

En general, se detecta que los usuarios del hashtag \#ArdeValencia suelen ser críticos con el Gobierno, presidido por el Partido Popular. Al respecto, no se registra ningún mensaje de apoyo a los líderes del PP junto a la etiqueta \#ArdeValencia. Cabe aclarar que durante los incendios, el PP pone en circulación otra etiqueta, \#todoscontraelfuego, utilizada por sus simpatizantes. No obstante, los partidos políticos de la oposición y los detractores de las políticas del PP emplean \#ArdeValencia. Así se observa en el tuit de @goriamarcosmar, excoordinadora de Izquierda Unida en la Comunidad Valenciana.



Además, cuando el espacio del tuit lo permite, se suelen añadir otros hashtags valorativos, como \#CastellanoDimisión, para pedir la dimisión del entonces consejero encargado de las políticas forestales en la Comunidad Valenciana, o \#MaiMés (\#NuncaMás), etiqueta que tuitean los dirigentes de la formación política Coalició Compromís y otros usuarios anónimos como @El_proscrito.

Paco Metal oel proscito i 8 de in de 2013

Vine por esa carretera. ambos lados quemados r ArdeVyuncia nMaimes

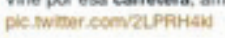

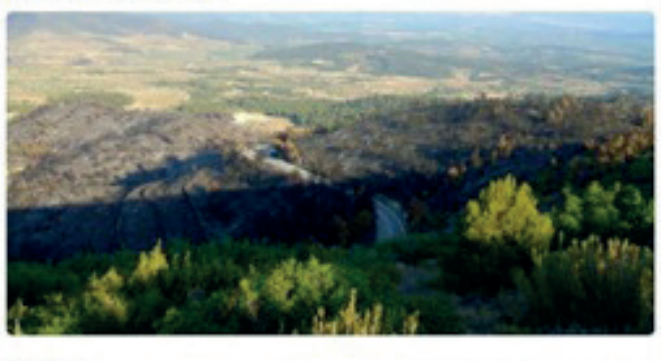




\section{Conclusiones}

Los resultados del análisis verifican las tres hipótesis planteadas al inicio de este artículo. Respecto a la primera, se confirma que en el caso \#ArdeValencia, referido a un suceso de actualidad imprevisto, los usuarios de hashtags emiten más valoraciones, en el $46,6 \%$ de los textos, que informaciones contrastadas, en el $39 \%$ de los tuits.

Además, cuando se valora, suele ser para cuestionar al Partido Popular y su gestión al frente de los gobiernos español y valenciano. Así ocurre en el $29,7 \%$ de los textos. Las críticas recaen sobre el presidente de España, Mariano Rajoy, en el $45 \%$ de las ocasiones. Desde esta perspectiva, se deduce el gusto de los usuarios por posicionarse ideológicamente, al menos, por apoyar a alguno de los bandos en liza. La mera elección del hashtag también puede implicar posicionamiento. En el supuesto concreto de \#ArdeValencia, la etiqueta se emplea con vehemencia entre partidos y políticos de la oposición. En general, se asocia a una visión negativa de la gestión del PP.

En relación con la segunda hipótesis, se confirma que el porcentaje de información exclusiva es escaso, solo presente en el 2,8\% de los textos, frente al elevado volumen de titulares publicados anteriormente por medios de comunicación tradicionales. Este argumento, respecto a la cobertura de los incendios, puede restar valor a Twitter como fuente informativa, pues se entiende que los profesionales encargados de informar sobre este suceso ya revisan los datos publicados por otros medios. No sería necesario recurrir a Twitter para conocer los detalles que difunde la competencia. Además, si las informaciones en circulación proceden de medios, pueden ser antiguas, como ocurre con los titulares de prensa escrita, que responden a datos conocidos el día anterior.

Por otro lado, las primicias divulgadas junto al hashtag \#ArdeValencia aluden a detalles poco relevantes. Por ejemplo, algunos usuarios anónimos asisten a la asamblea de vecinos damnificados e informan en la red social. No obstante, si bien es cierto que, en este estudio, Twitter apenas aporta datos exclusivos, puede funcionar como fuente informativa complementaria en determinados supuestos. Así, si el periodista interesado no cuenta con medios para desplazarse, puede consultar Twitter para seguir eventos en desarrollo, como la asamblea de damnificados.

Asimismo, dado que las exclusivas proceden de usuarios anónimos en el 35,7\% de los casos, una manera eficaz de localizarlas consiste en monitorizar todos los tuits asociados a hashtags que aluden al suceso, con independencia de si proceden de actores anónimos o identificados. Esta conclusión puede resultar interesante para los periodistas que acostumbren a evitar las publicaciones anónimas. Aunque las primicias se difundan de manera esporádica, como ocurre en el debate sobre \#ArdeValencia, parece aconsejable añadir la monitorización de hashtags a las rutinas periodísticas.

Respecto a la tercera hipótesis, se confirma la reiteración de informaciones y opiniones relacionadas con \#ArdeValencia, lo que disminuye la diversidad de significados. Resulta especialmente evidente en el supuesto de las críticas. La mayor parte puede reconducirse a dos ideas, cuestionar las prioridades del presidente Rajoy, que no visita la zona quemada, y denunciar los recortes del Gobierno valenciano en medios para la extinción de incendios.

En conclusión, los periodistas que siguen el hashtag \#ArdeValencia encuentran contada información exclusiva y poca diversidad de contenidos, normalmente ya publicados en los medios, además de una acentuada reiteración de opiniones similares. Por lo tanto, en este caso concreto, el alto grado de reiteración y la escasa exclusividad cuestionan la ideoneidad de Twitter como fuente informativa relevante. No obstante, para extrapolar esta conclu- 
sión, sería necesario ampliar la investigación y comparar los resultados de \#ArdeValencia con otros estudios de caso sobre sucesos imprevistos.

En contrapartida, la frecuente reiteración de contenidos muestra la utilidad de las etiquetas para maximizar la difusión. La incorporación del hashtag permite agrupar tuits que, en conjunto, contribuyen a crear un ambiente o estado de la cuestión sobre la gestión de los incendios por parte de las autoridades. Como se ha explicado, los usuarios incluyen la etiqueta al tiempo que manifiestan su posicionamiento respecto a las decisiones del Gobierno valenciano y los líderes del PP. También se detecta que los actores de \#ArdeValencia retuitean más valoraciones y críticas que informaciones, dato que podría justificar su interés por acudir a Twitter para conocer las opiniones de otros actores, incluso para secundarlas si las comparten.

En esta línea, puede afirmarse que el seguimiento de tuits vinculados a hashtags está en consonancia con el concepto de periodismo ambiental (Hermida, 2010), pues el volumen de mensajes y sus significados dejan entrever las tendencias que se adoptan en el debate, el sentir común de la comunidad virtual. Por encima de las ideas individuales, es posible percibir el ambiente que se respira en el debate de \#ArdeValencia, y si se producen cambios de tono o enfoque durante el proceso colectivo de toma de conciencia y formación de conocimiento (Hermida, 2010 \& 2013; Bruns \& Burgess, 2012).

Desde esta perspectiva, la reiteración de informaciones y valoraciones sirve de amplificador de las diferentes corrientes de opinión. Podría afirmarse que los usuarios de hashtags son emisores y receptores, fuente y audiencia de inclinaciones y preferencias. El seguimiento de hashtags puede suponer una fuente de pistas para los periodistas, sobre los intereses de la audiencia, si decaen o no dichos intereses, si es necesario profundizar en el tratamiento informativo o si es aconsejable cambiar de enfoque. Los hashtags sirven de puente entre cuentas y fomentan el crowdsourcing o elaboración colaborativa del ambiente o estado de la cuestión sobre el suceso de actualidad.

Además, el estudio de \#ArdeValencia evidencia que en Twitter se reproducen algunas tendencias que actualmente caracterizan la gestión de fuentes en los medios tradicionales. Por ejemplo, en Twitter también se intuye el intento de los políticos por controlar la agenda mediática (Hallin \& Mancini, 2004; Casero-Ripollés \& López-Rabadán, 2013) y se constata una importante presencia de fuentes oficiales e institucionales (García, 2010), que tuitean y se dirigen a sus potenciales votantes sin la mediación de la prensa. En relación a \#ArdeValencia, destaca el ejemplo de la formación política Coalició Compromís, muy activa durante el período analizado y promotora de la etiqueta \#MaiMés (\#NuncaMás).

En definitiva, aunque Twitter puede reproducir los vicios observados en las rutinas productivas de los medios convencionales, también permite abrir el debate a actores vetados en los foros oficiales, según se aprecia en este estudio, con una fuerte presencia de actores anónimos, pero también de profesionales expertos y colectivos ciudadanos, a los que el periodista podrá recurrir como informador, pero también como guardián de la información y curador de contenidos (Bhargava, 2009; Bruno, 2011; Malini, 2011; Hermida, 2012; McElroy, 2013; Cappeletti, 2014; Bakker, 2014), funciones que parece demandar la ingente circulación de datos.

\section{Referencias}

1. Abbagliatti, E. (2010). Curadores digitales y la otra biblioteca. Recuperado el 30 de julio de 2014, de http:// www.elmostrador.cl/opinion/2010/09/14/curadores-digitales-y-la-otra-biblioteca/ 
2. Abejón, P., Sastre, A. \& Linares, V. (2012). Facebook y Twitter en campañas electorales en España. Anuario Electrónico de Estudios en Comunicación Social "Disertaciones", 5 (1), 129-159.

3. Artwick, C. (2013). Reporters on Twitter. Digital Journalism, 1(2), 212-228. DOI: 10.1080/21670811. 2012.744555.

4. Bakker, P. (2014). Mr. Gates Returns. Journalism Studies. DOI: 10.1080/1461670X.2014.901783.

5. Bardin, L. (1996). Análisis de contenido. Madrid, España: Akal.

6. Bhargava, R. (2009) Manifiesto for the Content Curator: the next big Social Media job on the future? Recuperado el 30 de julio de 2014, de http//www.rohitbhargava.com/2009/09/manifiesto-for-thecontent-curator-the-next-big-social-media-job-of-the-future-html

7. Bradshaw, P. (2008). The chinese earthquake and Twitter - Crowdsourcing without managers. Online Journalism Blog. Recuperado el 5 de julio de 2014, de http://onlinejournalismblog.com/2008/05/12/ twitter-and-the-chineseearthquake/

8. Broersma, M., \& Graham, T. (2012). Social Media as Beat. Journalism Practice, 6 (3), 403-419. DOI:10.1080/ 17512786.2012 .663626$.

9. Brown-Smith, C. (2012). \#Memstorm: Twitter as a Community-driven Breaking News Reporting Tool. \#ISOJ Journal: The Official Journal of the International Symposium on Online Journalism, 2 (2), 5-28. Recuperado el 10 de julio de 2014, de https://online.journalism.utexas.edu/journals/ISOJ_Journal_V2_N2_2012_Fall.pdf

10. Bruno, N. (2011). Tweet First, Verify Later: how real-time information is changing the coverage of worldwide crisis events. Oxford: Reuters Institute for the Study of Journalism. Recuperado el 30 de julio de 2014, de http://nicolabruno.files.wordpress/2011/05/tweet/first_verify_later2.pdf

11. Bruns, A., \& Burgess, J. E. (2012). Researching news discussion on Twitter. Journalism Studies, 13 (5-6), 801814. DOI:10.1080/1461670X.2012.664428.

12. Burns, A. (2010). Oblique Strategies for Ambient Journalism. M/C Journal, 13(2). Recuperado el 28 de agosto de 2012, de http://journal.media-culture.org.au/index.php/mcjournal/article/view/230.

13. Cappelletti, M., \& Domínguez, S. (2014). La curaduría de contenidos y la narrativa colaborativa en el Ciberperiodismo: estudio del caso de Storify en el diario digital Elpais.com. Estudios sobre el Mensaje Periodístico, 20 (1), 17-31. Madrid, España. Servicio de Publicaciones de la Universidad Complutense.

14. Carrera Álvarez, P., Sainz de Baranda, C., Herrero, E., \& Limón, N. (2012). Journalism and Social Media: How Spanish Journalists Are Using Twitter. Estudios sobre el mensaje periodístico. Madrid, España. Servicio de Publicaciones de la Universidad Complutense.

15. Casero-Ripollés, A. (2009). El control político de la información periodística. Revista Latina de Comunicación Social, RLCS, 64, 354-366.

16. Casero-Ripollés, A. (2010). Prensa en Internet: nuevos modelos de negocio en el escenario de la convergencia. El Profesional de la Información, 19 (6), 595-601.

17. Casero-Ripollés, A., \& Feenstra, R. A. (2012). The 15M Movement and the New Media: A Case Study of How New Themes Were Introduced Into Spanish Political Discourse. Media International Australian, 144, 68-76.

18. Casero-Ripollés, A., \& López-Rabadán, P. (2013). La gestión de fuentes informativas como criterio de calidad profesional. En Gómez Mompart, J.L., Gutiérrez Lozano, J.F., \& Palau Sampio, D. (Ed.). La calidad periodística: teorías, investigaciones y sugerencias profesionales (pp. 73-89). Valencia, España: Aldea Global. 
19. Castells, M. (2009). Comunicación y poder. Madrid, España. Alianza Editorial.

20. Castells, M. (2012). Networks of Outrage and Hope: Social Movements in the Internet Age. Cambridge: Polity.

21. Della Porta, D. (2011). Communication in movement: Social movements as agents of participatory democracy. Information Communication and Society, 14 (6), 800-819.

22. Deltell, L., Congosto, M. L., Claes, F., \& Osteso, J. M. (2013). Identificación y análisis de los líderes de opinión en Twitter en torno a Hugo Chávez. Revista Latina de Comunicación Social, RLCS, 68, 696-718.

23. Deuze, M. (2005). What Is Journalism? Professional identity and ideology of journalists reconsidered. Journalism, 6, 442-464.

24. Edo, C., \& Mayoral, J. (2011). El incipiente desarrollo del ciberperiodismo audiovisual. Análisis de ocho cibermedios españoles. Presentado en el III Congreso Internacional de Ciberperiodismo y Web 2.0. La transformación del espacio mediático, 206-226. Bilbao, España: Universidad del País Vasco

25. Farhi, P. (2009). The Twitter Explosion, American Journalism Review, 31(3), 26-31. Recuperado el 10 de julio de 2014, de http://www.ajr.org/article.asp?id=4756

26. Farhi, P. (2011, 12 de abril). NPR's Andy Carvin, Tweeting the Middle East. The Washington Post. Recuperado el 10 de julio de 2011, de http://www.washingtonpost.com/lifestyle/style/npr-andy-carvin-tweeting-themid-\%20dle-east/2011/04/06/AFcSdhSD_story.html

27. Feenstra, R. A., \& Casero-Ripollés, A. (2014). Democracy in the Digital Communication Environment: A Typology Proposal of Political Monitoring Processes. International Journal of Communication, 8, 24482468.

28. Flores, J.M. (2009). Nuevos modelos de comunicación, perfiles y tendencias de las redes sociales. Comunicar, 33, 73-81.

29. Franklin, B. (2011). Sources, Credibility and the Continuing Crisis of UK Journalism. En Franklin, B., \& Carlson, M. (Ed.). Journalism, Sources and Credibility. New Perspectives (pp. 90-106). Londres, Inglaterra: Routledge.

30. Gans, H. J. (1979). Deciding What's News. A Study of CBS Evening News, NBC Nightly News and Time. Nueva York, Estados Unidos: Pantheon Books.

31. García-Avilés, J. A., Kaltenbrunner, A., \& Meier, K. (2014). Media Convergence Revisited. Journalism Practice. DOI:10.1080/17512786.2014.885678.

32. García de Torres, E., et ál. (2011). See you on Facebook or Twitter? The Use of Social Media by 27 News Outlets from 9 Regions in Argentina, Colombia, Mexico, Peru, Portugal, Spain and Venezuela. Presentado en el $12^{\circ}$ International Symposium on Online Journalism. Austin, Texas.

33. García-Santamaría, J. V. (2010). Crisis del periodismo de fuentes. Las prácticas del periodismo en España en el accidente de Spanair. Revista Latina de Comunicación Social, RLCS, 65, 516-537.

34. Goyanes, M. (2014). An Empirical Study of Factors that Influence the Willingness to Pay for Online News. Journalism Practice. DOI: 10.1080/17512786.2014.882056.

35. Greer, C., \& Ferguson, D. (2011). Using Twiter for Promotion and Branding: a Content Analysis of Local Television Twitter Sites. Journal of Broadcasting and Electronic Media, 55 (2), 198214.

36. Grossi, G. (2007). La opinión pública. Madrid, España: CIS. 
37. Hallin, D., \& Mancini, P. (2004). Comparing Media Systems: Three Models of Media and Politics. Nueva York, Estados Unidos: Cambridge University Press.

38. Hermida, A. (2010). Twittering the news: The emergence of ambient journalism. Journalism Practice, 4 (3), 297-308. DOI:10.1080/17512786.2012.667269.

39. Hermida, A. (2012). Tweets and truth. Journalism Practice, 6 (5-6), 659-668. DOI:10.1080/17512786.2012.6 67269.

40. Hermida, A. (2013). \#Journalism. Digital Journalism, 1 (3), 295-31. DOI: 10.1080/21670811.2013.808456.

41. Herrero, E. (2012). Fuentes periodísticas y redes sociales en las noticias de Marta del Castillo. Estudios sobre el Mensaje Periodístico, 19 (1), 453470. Madrid, España: Universidad Complutense.

42. Hu, M., Liu, S., Wei, F., et ál. (2012). Breaking News on Twitter. Presentado en el $13^{\circ}$ International Symposium on Online Journalism. Austin, Texas. Recuperado el 12 de agosto de 2013, de http://www. cc.gatech.edu/ stasko/papers/chi12-twitter.pdf

43. Krippendorf, K. (1990). Metodología de análisis de contenido. Barcelona, España: Paidós.

44. Kwak, H., Lee, Ch., Park, H., et ál. (2010). What Is Twitter, a Social Network or a News Media. Recuperado el 12 de agosto de 2012, de http://product.ubion.co.kr/upload20120220142222731/ccres00056/db/_2250_1/ embedded/2010-www-twitter.pdf

45. Lasorsa, D., Lewis, S., \& Holton, A. (2012). Normalizing Twitter. Journalism Studies, 13 (1), 19-36. DOI:10.1 080/1461670X.2011.571825.

46. López, X., \& Alonso, S. (2013). Los periódicos gallegos en las redes sociales virtuales: presencia y posicionamiento en el nuevo escenario comunicativo. Estudios sobre el Mensaje Periodístico, 19 (2), 1001 1016. Madrid, España: Universidad Complutense

47. Malini, F. L. (2011). Cobertura colaborativa nas redes sociais: entre a emergência e a programação. Recuperado el 30 de julio de 2014, de http://fabiomalini.com/o-que-e-ser-jornalista-hoje/coberturacolaborativa-entre-a-programacao-e-a-emergencia/

48. McElroy, K. (2013). Where Old (Gatekeepers) Meets New (Media). Journalism Practice, 7 (6), 755-771. DOI: 10.1080/17512786.2013.774117.

49. Micó, J. LL. (2011). El futuro para los graduados en periodismo en el entorno digital. Presentado en el III Congreso Internacional de Ciberperiodismo y Web 2.0. La transformación del espacio mediático, 86-97. Bilbao, España: Universidad del País Vasco50.

50. Micó, J. LL., \& Casero-Ripollés, A. (2013). Political Activism Online: Organization and Media Relations in the Case of 15M in Spain. Information, Communication and Society. DOI: 10.1080/1369118X.2013.830634.

51. Newman, N. (2009). The Rise of Social Media and its Impact on Mainstream Journalism. Oxford: Reuters Institute for the Study of Journalism. University of Oxford. Recuperado el 12 de agosto de 2012, de http:// www.sssup.it/UploadDocs/6635_8_S_The_rise_of_Social_Media_and_its_Impact_on_mainstream_ journalism_Newman_07.pdf

52. Newman, N., Dutton, W., \& Blank, G. (2012). Social Media in the Changing Ecology of News: The Fourth and Fifth Estates in Britain. International Journal of Internet Science, 7 (1), 6-22. Recuperado el 10 de Julio de 2014, de http://www.ijis.net/ijis7_1/ijis7_1_newman_et_al.pdf 
53. Niles, R. (2007). A Journalist's Guide to Crowdsourcing. OJR: The Online Journalism Review. Recuperado el 10 de septiembre de 2012, de http://www.ojr.org/ojr/stories/070731niles

54. Noguera, J. M. (2013). How Open are Journalists in Twitter? Trends Towards the End User Journalism. Comunicación y Sociedad, 26 (1), 93114.

55. Nunomuro, E. (2013). A imprensa, o Twitter e as eleições de 2010 no Brasil. Intercom - RBCC. 36 (1), 103126. São Paulo, Brasil.

56. Oriella PR Network (2012). The Influence Game: How News is Sourced and Managed Today. The Oriella PR Network Global Digital Journalism Study, 2012.

57. Oriella PR Network (2013). The New Normal for News: Have Global Media Changed Forever? The Oriella PR Network Global Digital Journalism Study, 2013.

58. Orihuela, J. L. (2011). 80 claves sobre el futuro periodismo. Madrid, España: Anaya Multimedia.

59. Ortells-Badenes, S., \& González-Molina, S. (2012, octubre). La polivalencia periodística de los profesionales en las redes sociales. Estudios sobre el Mensaje Periodístico, 18, (núm. especial), 455463. Madrid, España: Universidad Complutense.

60. Phillips, A. (2010). New Sources: Old Bottles. En Fenton, N. (Ed.). New Media, Old News. Journalism and Democracy in the Digital Age (pp. 87-101). Londres, Inglaterra: Sage.

61. Requejo, J. L., \& Herrera, S. (2014). Retroceso en la creación de comunidad entre los diarios generalistas españoles en Twitter (2011 2013). Estudios sobre el Mensaje Periodístico, 20 (1), 229248. Madrid, España: Universidad Complutense.

62. Rodríguez, A., \& García López, J. (2013, abril). Uso y funciones de Twitter en periodistas españoles. Estudios sobre el mensaje periodístico, 19, (núm. especial), 963969. Madrid, España: Universidad Complutense.

63. Rojo, R. (2012). Existe alguna relación entre la audiencia televisiva y la cantidad de tweets sobre un programa: el caso de Mundos Opuestos. (Tesis Doctoral). Santiago de Chile. Facultad de Economía y Negocios. Universidad de Chile.

64. Romero, D., Meeder, B., \& Kleinberg, J. (2011). Differences in the Mechanics of Information Diffusion Across Topics: Idioms, Political Hashtags, and Complex Contagion on Twitter. Presentado en Proceedings of 20th ACM International World Wide Web Conference de Hyderabad, India. Recuperado el 28 de junio de 2012, de http://www.cs.cornell.edu/home/kleinber/www.11-hashtags.pdf

65. Rubio García, R. (2014). Twitter \& la teoría de la Agenda-Setting: mensajes de la opinión pública digital. Estudios sobre el Mensaje Periodístico, 20 (1), 249264. Madrid, España. Servicio de Publicaciones de la Universidad Complutense.

66. Salaverría, R., García-Avilés, J. A., \& Masip, P. (2010). Concepto de convergencia periodística. En López, X. \& Pereira, X. (coords.). Convergencia Digital. Reconfiguración de los Medios de Comunicación en España (pp. 4164). Santiago de Compostela, España: Universidad de Santiago de Compostela.

67. Said Hung, E., Serrano Tellería, A., García de Torres, E., Yerezers'ka, L., \& Calderín, M. (2013). La gestión de los Social Media en los medios informativos iberoamericanos. Comunicación y Sociedad, 26 (1), 67-92.

68. Serrano, P. (2009). Desinformación. Cómo los medios ocultan el mundo. Madrid, España: Editorial Península.

69. Serrano, P. (2013). La comunicación jibarizada. Como la tecnología ha cambiado las mentes. Madrid, España: Editorial Península. 
70. Shirky, C. (2011). A cultura da participação: criatividade e generosidade no mundo conectado. Zahar, 31 (5). Rio de Janeiro, Brasil.

71. Singer, J. B. (2011). Crowd Control: Collaborative Gatekeeping in a Shared Media Space. Presentado para Newspaper Division Association for Education in Journalism and Mass Communication. Recuperado el 14 de julio de 2014, de http://citation.allacademic.com//meta/p_mla_apa_research_citation/5/2/0/3/3/ pages520338/p520338-1.php

72. Soengas, X. (2007). Los vínculos entre la información y el poder en la sociedad actual. Icono, 14 (12), 292-322.

73. Tuchman, G. (1983). La producción de la noticia. Estudio sobre la construcción de la realidad. Barcelona, España: Gustavo Gili.

74. Vis, F. (2013). Twitter As a Reporting Tool for Breaking News. Digital Journalism, 1 (1), 27-47. DOI: 10.1080/21670811.2012.741316.

75. Wolf, M. (1994). Los efectos sociales de los media. Barcelona, España: Paidós. 